\title{
PROMOTING EXTERNAL INOSCULATION OF PREVASCULARISED TISSUE CONSTRUCTS BY PRE-CULTIVATION IN AN ANGIOGENIC EXTRACELLULAR MATRIX
}

\author{
M.W. Laschke ${ }^{1,2, *}$, H. Mussawy ${ }^{1,2}$, S. Schuler ${ }^{1,2}$, D. Eglin ${ }^{3}$, M. Alini ${ }^{3}$, and M.D. Menger ${ }^{1,2}$ \\ ${ }^{1}$ Institute for Clinical \& Experimental Surgery, University of Saarland, D-66421 \\ Homburg/Saar, Germany \\ ${ }^{2}$ Collaborative Research Center AO Foundation, University of Saarland, D-66421 \\ Homburg/Saar, Germany \\ ${ }^{3}$ AO Research Institute Davos, Clavadelerstrasse, CH-7270 Davos Platz, Switzerland
}

\begin{abstract}
The engineering of preformed microvessels offers the promising opportunity to rapidly vascularise implanted tissue constructs by the process of inosculation. Herein, we analyzed whether this process may further be accelerated by cultivation of prevascularised tissue constructs in Matrigel before implantation. Nano-size hydroxyapatite particles/poly(ester-urethane) scaffolds were implanted into the flank of FVB/N-TgN (Tie2/GFP) 287 Sato mice to allow the ingrowth of a granulation tissue with green fluorescent protein (GFP)-positive blood vessels. After harvesting, these prevascularised constructs were then transferred into dorsal skinfold chambers of $\mathrm{FVB} / \mathrm{N}$ recipient mice to study the process of inosculation. The constructs were implanted directly after embedding in Matrigel or after 3 days of cultivation in the extracellular matrix. Matrigel-free constructs served as control. Cultivation in Matrigel resulted in the outgrowth of CD31/GFP-positive vascular sprouts. Vascularisation of these constructs was markedly improved when compared to the other two groups, as indicated by a significantly elevated functional microvessel density between days 6 to 14 after implantation into the dorsal skinfold chamber. This was associated with an increased number of GFP-positive blood vessels growing into the surrounding host tissue. Thus, the blood supply to prevascularised tissue constructs can be accelerated by their pre-cultivation in an angiogenic extracellular matrix, promoting external inosculation of the preformed microvascular networks with the host microvasculature.
\end{abstract}

Keywords: Tissue engineering, inosculation, scaffold, Matrigel, vascularisation, angiogenesis, dorsal skinfold chamber, intravital fluorescence microscopy.

\author{
*Address for correspondence: \\ Matthias W. Laschke \\ Institute for Clinical \& Experimental Surgery \\ University of Saarland \\ D-66421 Homburg/Saar \\ Germany
}

Telephone Number: +496841 1626554

FAX Number: +4968411626553

E-mail: matthias.laschke@uniklinik-saarland.de

\section{Introduction}

The rapid vascularisation of implanted tissue constructs represents a major prerequisite for the successful introduction of tissue engineering applications to clinical practice (Laschke et al., 2006; Rouwkema et al., 2008; Lovett et al., 2009). A promising strategy to achieve this goal is the engineering of tissue substitutes with preformed microvascular networks, which are capable of inosculating with the host microvasculature after implantation (Lokmic and Mitchell, 2008; Laschke et al., 2009). By this, it may be possible to rapidly establish a sufficient blood supply in the centre of large three-dimensional tissue constructs, avoiding the time-consuming ingrowth of new blood vessels into the implants via the process of angiogenesis. Nonetheless, several studies indicate that even this approach cannot guarantee an adequate blood perfusion of the constructs during the very first days after implantation (Tremblay et al., 2005; Laschke et al., 2008a). Therefore, novel strategies have to be established, which accelerate the interconnection between preformed microvascular networks within implanted tissue constructs and blood vessels of the surrounding host tissue.

There are two principal modes of inosculation, i.e. internal and external inosculation (Laschke et al., 2009). Internal inosculation occurs inside a prevascularised tissue graft when the preformed microvessels regress and microvessels of host origin invade the graft along the previously patterned vascular channels (Capla et al., 2006). It is, however, also possible that the preformed microvessels grow out of prevascularised tissue constructs, resulting in external inosculation in the surrounding host tissue (Laschke et al., 2008a). The latter may in particular be observed when the preformed microvascular network exhibits a high sprouting angiogenic activity. In the present study we analyzed whether this may be induced by cultivation of prevascularised tissue constructs in an angiogenic extracellular matrix before implantation. Our hypothesis was that the in vitro outgrowth of vascular sprouts into the extracellular matrix may promote external inosculation after implantation and, thus, may accelerate the restoration of blood perfusion in the implants.

For our study, we pre-cultivated in situ generated prevascularised tissue constructs in Matrigel. This sterile extract of basement membrane proteins forms a 3D gel at $37^{\circ} \mathrm{C}$ and contains a combination of different angiogenic growth factors, which stimulate the development of blood 
vessels (Nicosia and Ottinetti, 1990; Kleinmann and Martin, 2005).

\section{Materials and Methods}

\begin{abstract}
Animals
For our study we used 12- to 16-week-old transgenic FVB/ N-TgN (Tie2/GFP) 287 Sato mice (The Jackson Laboratory, Maine, USA) and corresponding FVB/N wildtype mice (Charles River, Sulzfeld, Germany) with a body weight of $22-25 \mathrm{~g}$. The animals were housed one per cage and had free access to tap water and standard pellet food (Altromin, Lage, Germany). All experiments were approved by the local governmental animal care committee and were conducted in accordance with the German legislation on protection of animals and the NIH Guidelines for the Care and Use of Laboratory Animals (NIH Publication \#85-23 Rev. 1985).
\end{abstract}

\section{Experimental protocol}

For the in vivo experiments, 23 poly(ester-urethane) scaffolds were implanted into the flank of $6 \mathrm{FVB} / \mathrm{N}-\mathrm{TgN}$ (Tie2/GFP) 287 Sato mice for 20 days to create in situ prevascularised tissue constructs. These constructs were implanted into the dorsal skinfold chamber of $\mathrm{FVB} / \mathrm{N}$ recipient mice directly after embedding in Matrigel $(n=7)$ or after 3 days of cultivation in the extracellular matrix $(n=8)$, whereas Matrigel-free constructs $(n=8)$ served as control. Intravital fluorescence microscopy of vascularisation, inosculation and microhaemodynamics was performed immediately as well as 3, 6, 10 and 14 days after construct implantation. At the end of the in vivo experiments, the animals were sacrificed with an overdose of the anaesthetic and the dorsal skinfold preparations were excised for further immunohistochemical analyses.

\section{Preparation of in situ prevascularised tissue constructs}

Porous nano-size hydroxyapatite particles/poly(esterurethane) composite scaffolds with a size of $\sim 3 \times 3 \times 1 \mathrm{~mm}$ (Fig. 1a) were used for the generation of in situ prevascularised tissue constructs. These scaffolds, which exhibit an excellent in vivo biocompatibility, were fabricated by a salt leaching-phase inverse process, as described previously in detail (Laschke et al., 2010). In a first step, the scaffolds were implanted for 20 days into the flank of transgenic FVB/N-TgN (Tie2/GFP) 287 Sato mice to allow the ingrowth of a granulation tissue with green fluorescent protein (GFP)-positive microvessels (Fig. 1b) (Motoike et al., 2000). For this purpose, the animals were anesthetized by i.p. injection of ketamine (75 mg/kg body weight; Pharmacia GmbH, Erlangen, Germany) and xylazine 2\% (25 mg/kg body weight; Rompun, Bayer, Leverkusen, Germany) and four scaffolds per mouse were implanted into subcutaneous pockets of the right and left flank. After 20 days, the in situ generated prevascularised tissue constructs were carefully excised and embedded in 200 $\mu$ l Matrigel (BD Matrigel ${ }^{\mathrm{TM}}$ Matrix; BD Biosciences, Heidelberg, Germany) at the bottom of
$35 \mathrm{~mm}$ Petri dishes. The Matrigel was allowed to polymerize for $30 \mathrm{~min}$ at $37^{\circ} \mathrm{C}$ and $5 \% \mathrm{CO}_{2}$. Subsequently, the Petri dishes were filled with $2 \mathrm{~mL}$ of $37^{\circ} \mathrm{C}$ warm Dulbecco's modified Eagle's medium (DMEM; $10 \%$ foetal calf serum, $100 \mathrm{U} / \mathrm{mL}$ penicillin, $0.1 \mathrm{mg} / \mathrm{mL}$ streptomycin; PAA, Cölbe, Germany) and the Matrigel-embedded prevascularised tissue constructs were cultivated for 3 days to stimulate the outgrowth of tubular vessel-like structures (Figs. 1c and d). For the implantation into dorsal skinfold chambers of FVB/N wild-type mice, the tissue constructs were then cut out of the Matrigel with a scalpel, taking care to maintain the small margin of Matrigel, which contained outgrowing vascular sprouts. In a second group of animals, the constructs were implanted into dorsal skinfold chambers directly after embedding in Matrigel without pre-cultivation. Matrigel-free tissue constructs, which were transferred directly from the flank of transgenic FVB/N-TgN (Tie2/GFP) 287 Sato mice into dorsal skinfold chambers of wild-type animals, served as controls.

\section{Preparation of the dorsal skinfold chamber and construct implantation}

Inosculation and vascularisation of prevascularised tissue constructs were analyzed within dorsal skinfold chambers of FVB/N wild-type mice to distinguish between GFPpositive preformed blood vessels of the implanted tissue constructs and GFP-negative blood vessels of the host tissue within the chamber (Fig. 1e). The chamber preparation has been described previously in detail (Menger et al., 2002). Briefly, the mice were anaesthetised by i.p. injection of ketamine $(75 \mathrm{mg} / \mathrm{kg}$ body weight; Pharmacia $\mathrm{GmbH})$ and xylazine $2 \%(25 \mathrm{mg} / \mathrm{kg}$ body weight; Rompun, Bayer). Subsequently, two symmetrical titanium frames were implanted on the extended dorsal skinfold of the animals, so that they sandwiched the double layer of skin. One layer of skin was then completely removed in a circular area of $\sim 15 \mathrm{~mm}$ in diameter, and the remaining layers, consisting of striated skin muscle, subcutaneous tissue and skin, were covered with a removable coverslip incorporated into one of the titanium frames. After the preparation, the animals were allowed to recover from anaesthesia and surgery for $72 \mathrm{~h}$ to exclude alterations of the microcirculation due to the surgical trauma.

For the implantation of the tissue constructs, the cover glass of the dorsal skinfold chamber was temporarily removed and the constructs were placed onto the striated muscle tissue within the centre of each chamber, taking care to avoid contamination, mechanical irritation or damage of the tissue.

\section{Intravital fluorescence microscopy and microcirculatory analysis}

For intravital fluorescence microscopy $0.05 \mathrm{ml} 5 \%$ fluorescein isothiocyanate (FITC)-labelled dextran 150,000 (Sigma; Deisenhofen, Germany) was given i.v. via the retrobulbary space. This provided contrast enhancement of the perfused microvasculature by staining of blood plasma. The dorsal skinfold chamber was then attached to the microscopic stage. Microscopy was 

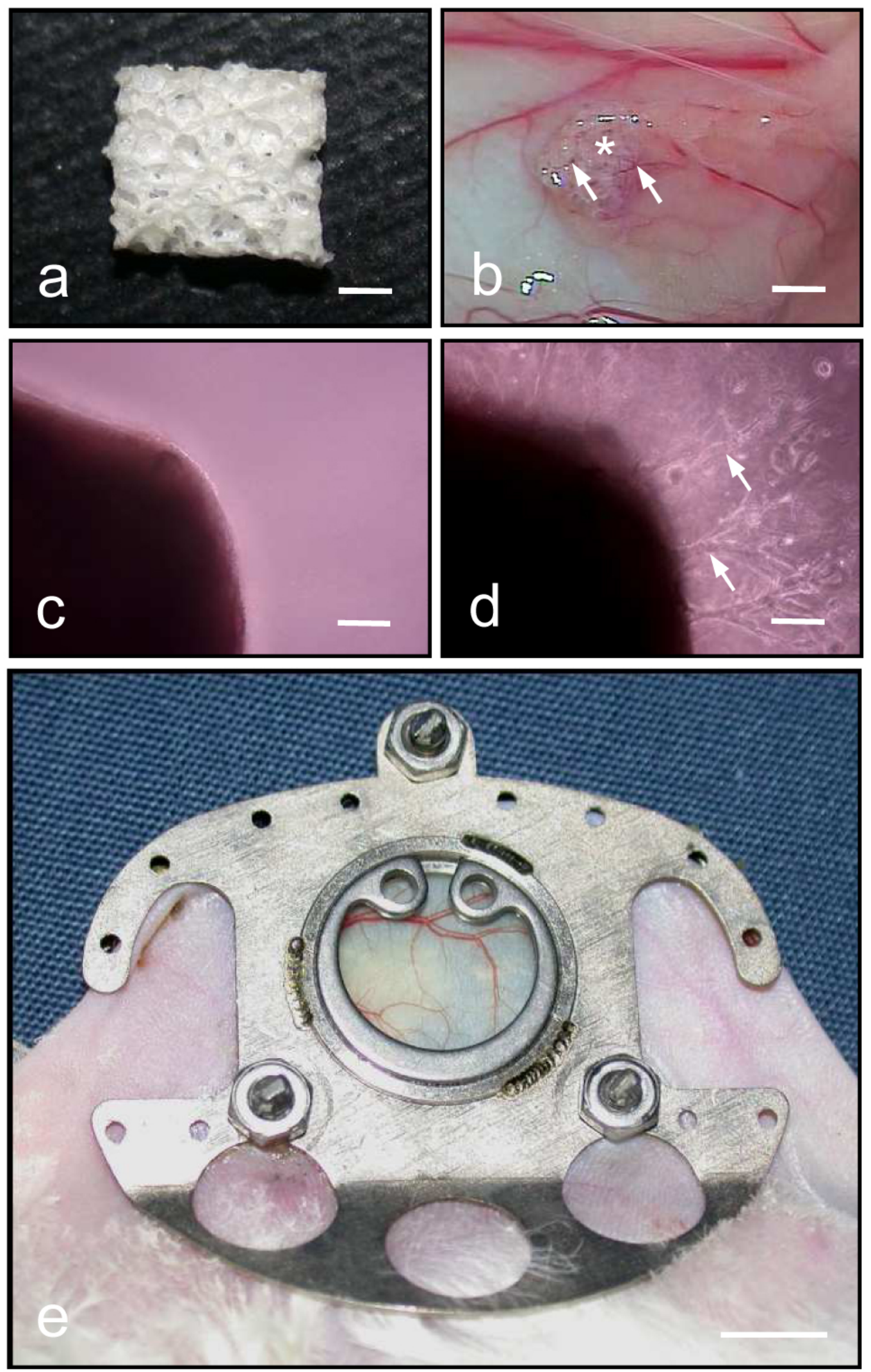

Fig. 1: (a) Porous nano-size hydroxyapatite particles/poly(ester-urethane) composite scaffold for the generation of in situ prevascularised tissue constructs. (b) For in situ prevascularisation, the scaffold (asterisk) was implanted into the flank of a FVB/N-TgN (Tie2/GFP) 287 Sato mouse. After 20 days, microvessels (arrows) from the surrounding host microvasculature had grown into the scaffold pores, resulting in a well vascularised tissue construct. c, d: Border of a prevascularised tissue construct directly after embedding in Matrigel (c) and after 3 days of cultivation in Matrigel (d), which results in the outgrowth of tubular vessel-like structures (d, arrows) into the extracellular matrix. (e) Dorsal skinfold chamber of a FVB/N wild-type mouse, which served as the host site for the implantation of prevascularised tissue constructs to analyze in vivo the process of inosculation using intravital fluorescence microscopy. Scale bars: (a) $=800 \mu \mathrm{m} ;(\mathbf{b})=1.7 \mathrm{~mm} ; \mathbf{( c , ~ d ) ~}=100 \mu \mathrm{m} ;(\mathbf{e})=5.2 \mathrm{~mm}$. 

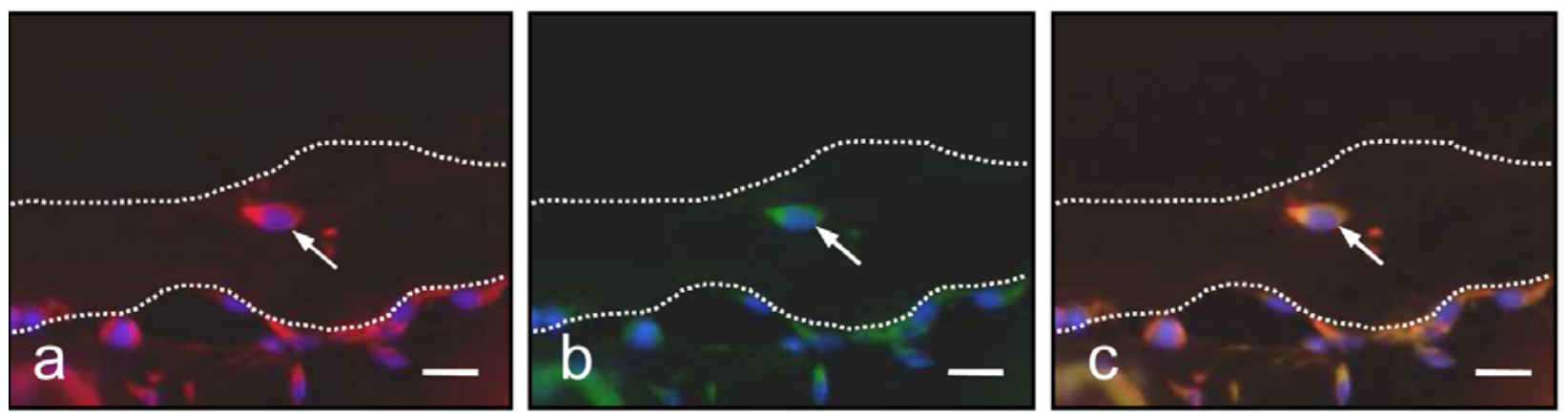

Fig. 2. (a-c) Immunohistochemical detection of a CD31/GFP-positive endothelial cell (arrow), which migrated into the Matrigel (border marked by broken lines), surrounding a prevascularised tissue construct 3 days after harvesting from the flank of a FVB/N-TgN (Tie2/GFP) 287 Sato mouse and cultivation in the extracellular matrix. Histological sections were stained with Hoechst to identify cell nuclei (a-c, blue), an antibody against CD31 for the detection of endothelial cells (a, red) and an antibody against GFP (b, green). (c) displays a merge of (a) and (b). Scale bars: (ac) $=14 \mu \mathrm{m}$.

performed using a Zeiss Axiotech microscope (Zeiss, Oberkochen, Germany) with a $100 \mathrm{~W}$ mercury lamp attached to an epi-illumination filter block for blue, green and ultraviolet light. The microscopic images were recorded by a charge-coupled device video camera (FK6990, Pieper, Schwerte, Germany) and transferred to a DVD system for off-line evaluation. By means of 5x, $10 \mathrm{x}$ and $20 \mathrm{x}$ long-distance objectives (Zeiss) magnifications of $x 115, x 230$ and $x 460$ were achieved on a 14 inch video screen (KV-14CT1E, Sony, Tokyo, Japan).

Quantitative off-line analysis of the microscopic images was performed using the software package CapImage (Zeintl, Heidelberg, Germany). Vascularisation of the tissue constructs was analyzed at a magnification of $x 460$ in 8 different microvascular regions of interest (ROIs) in the border zone and 8 different microvascular ROIs in the centre of the implants. Perfused ROIs were defined as areas, which exhibited either newly developed red blood cell (RBC)-perfused microvessels or preformed reperfused microvessels. In addition, functional capillary density, i.e. the length of all RBC-perfused blood vessels per observation area, was measured and is given in $\mathrm{cm} / \mathrm{cm}^{2}$. To study the process of inosculation, 10-15 preformed microvessels were randomly chosen in each prevascularised tissue construct at the day of implantation. During the further observation time points, the number of these vessels, which presented with reperfusion, was assessed and is given as percent of all analyzed vessels.

Diameters, centreline velocity, volumetric blood flow, and wall shear rate were determined in 10-15 randomly chosen microvessels within the centre zone of each scaffold. Diameters (d) were measured in $\mu \mathrm{m}$ perpendicularly to the vessel path. Centreline red blood cell velocity (v) was analyzed by CapImage using the line shift method. Volumetric blood flow was calculated by Q $=\pi *(\mathrm{~d} / 2)^{2} * \mathrm{v} / 1.6[\mathrm{pl} / \mathrm{s}]$, where 1.6 represents the BakerWayland factor (1974) to correct for the parabolic velocity profile in microvessels. Finally, wall shear rate (y) was calculated based on the Newtonian definition: $y=8 * v / d$.

\section{Immunohistochemistry}

For immunohistochemical detection of GFP-positive and -negative microvessels in the border zones of the implanted tissue constructs, paraffin-embedded $5 \mu \mathrm{m}$-thick sections were stained with a monoclonal rat-anti-mouse antibody against CD31 (1:30; Dianova GmbH, Hamburg, Germany) to detect endothelial cells and with a goat-anti-GFP antibody (1:200; Biomol, Hamburg, Germany) to enhance GFP-fluorescence. As secondary antibodies a goat-antirat $\mathrm{Cy} 3$ antibody (1:50; Dianova $\mathrm{GmbH})$ and a biotinlabeled donkey-anti-goat antibody (1:15; Jackson ImmunoResearch, Baltimore, MD, USA), which was detected by fluorescein labeled-streptavidin (1:50; Vector Labs, Burlingame, CA, USA), were used. For this purpose, the sections were placed in Coplin jars with $0.05 \%$ citraconic anhydride solution ( $\mathrm{pH}$ 7.4) for $1 \mathrm{~h}$ at $98^{\circ} \mathrm{C}$ and then incubated overnight at $4^{\circ} \mathrm{C}$ with the first antibody, followed by the appropriate secondary antibody at $37^{\circ} \mathrm{C}$ for $1 \mathrm{~h}$. On each section, cell nuclei were stained with Hoechst (1:500; Sigma) to merge the images exactly. For the quantitative analysis of GFP-positive microvessels (\%) in the border zones of the implanted tissue constructs, the sections were examined using a BX60 microscope (Olympus, Hamburg, Germany).

\section{Statistics}

After testing the data for normal distribution and equal variance, differences between the three study groups were analyzed by ANOVA followed by the appropriate post hoc comparison. To test for time effects in the individual groups, ANOVA for repeated measures was applied. This was followed by the paired Student's $t$-test, including correction of the $\alpha$-error according to Bonferroni probabilities for repeated measurements (SigmaStat; Jandel Corporation, San Rafael, CA, USA). All values are expressed as means \pm SEM. Statistical significance was accepted for a value of $P<0.05$. 


\section{Results}

\section{Angiogenic activation of prevascularised tissue constructs in vitro}

Pre-cultivation of in situ generated prevascularised tissue constructs for 3 days in Matrigel resulted in the outgrowth of tubular vessel-like structures into the extracellular matrix (Fig. 1d). This indicates the onset of sprouting angiogenic activity of the preformed microvascular networks. Immunohistochemical analysis further confirmed that CD31/GFP-positive endothelial cells migrated into the Matrigel margin surrounding the tissue constructs (Fig. 2).

\section{Vascularisation of implanted tissue constructs}

After implantation of the prevascularised tissue constructs into the dorsal skinfold chamber of FVB/N wild-type mice, perfused ROIs could be observed at the constructs' border zones of all three experimental groups already at day 3 (Fig. 3g). At this time point, haemorrhages were found around the tissue constructs (Figs. 3a-c). This was particularly the case in the group of constructs, which had been pre-cultivated for 3 days in Matrigel before implantation (Fig. 3c). Throughout the further observation period, the number of perfused microvessels rapidly increased in the border and centre zones of the tissue constructs, finally amounting to $100 \%$ of perfused ROIs at day 14 (Figs. 3d-h). Interestingly, pre-cultivation of tissue constructs in Matrigel markedly accelerated this vascularisation process, as indicated by a significantly increased number of perfused ROIs in the implant centre at day 6 when compared to the other two groups (Fig. 3h).
Accordingly, Matrigel-cultivated tissue constructs further exhibited a significantly increased functional microvessel density in the border and centre zones between days 6 and 14 in comparison to non-cultivated and Matrigel-free control implants (Figs. 3d-f and i-j).

\section{Haemodynamics of microvessels within implanted tissue constructs}

Matrigel-free control constructs exhibited constant microvessel diameters of 19-21 $\mu \mathrm{m}$ between day 6 and 14 after implantation into the dorsal skinfold chamber (Tab. 1). In contrast, Matrigel-embedded constructs presented with a microvessel diameter of $18 \mu \mathrm{m}$ at day 6 , which decreased to $12 \mu \mathrm{m}$ until the end of the 2 -weeks observation period, independently on whether they had been precultivated or not (Table 1).

In all three groups, the microvessels' centreline velocity progressively increased from $61-83 \mu \mathrm{m} / \mathrm{s}$ at day 6 to 145 $166 \mu \mathrm{m} / \mathrm{s}$ at day 14 (Table 1). Notably, Matrigel-cultivated tissue constructs exhibited a significantly increased centreline velocity at day 10 when compared to controls, indicating an accelerated restoration of blood perfusion after implantation into the dorsal skinfold chamber.

Both groups of Matrigel-embedded tissue constructs presented with a significantly decreased volumetric blood flow at day 14 when compared to the control group (Table 1). Because the calculation of volumetric blood flow is based on the diameter and centreline velocity of the analyzed microvessels, this result can clearly be attributed to the observed decrease of microvessel diameters in these groups. Vice versa, wall shear rates in both groups of Matrigel-embedded tissue constructs progressively

Table 1. Diameter $(\mu \mathrm{m})$, centreline velocity $(\mu \mathrm{m} / \mathrm{s})$, volumetric blood flow $(\mathrm{pL} / \mathrm{s})$ and wall shear rate $\left(\mathrm{s}^{-1}\right)$ of perfused blood vessels within in situ prevascularised tissue constructs at day 3, 6, 10 and 14 after implantation into dorsal skinfold chambers, as assessed by intravital fluorescence microscopy and computer-assisted image analysis. The constructs were implanted into the chamber directly after embedding in Matrigel (Matrigel) or after 3 days of precultivation in the extracellular matrix ( $3 \mathrm{~d}$ Matrigel). Matrigel-free constructs served as controls (Control).

Diameter $[\mu \mathrm{m}]$ :
Control
Matrigel
3d Matrigel
Centreline velocity $[\mu \mathrm{m} / \mathrm{s}]$ :
Control
Matrigel
3d Matrigel
Volumetric blood flow $[\mathrm{pL} / \mathrm{s}]$ :
Control
Matrigel
3d Matrigel
Wall shear rate $\left[\mathrm{s}^{-1}\right]$ :
Control
Matrigel
3d Matrigel

d3

d6

-- $\quad 20.7 \pm 1.5$

-- $\quad 17.5 \pm 2.2$

-- $\quad 17.5 \pm 0.6$

-- $\quad 60.5 \pm 8.1$

$\begin{array}{ll}-- & 62.8 \pm 25.0\end{array}$

$--\quad 83.1 \pm 8.8$

-- $\quad 46.9 \pm 11.9$

$--\quad 16.1 \pm 5.5$

-- $\quad 36.2 \pm 5.8$

-- $\quad 25.3 \pm 3.1$

$--\quad 40.3 \pm 17.3$

-- $\quad 42.0 \pm 4.4$ d10

$19.8 \pm 1.2$

$14.9 \pm 1.7^{*}$

$13.2 \pm 1.0^{\mathrm{a} *}$

$107.7 \pm 9.1^{\mathrm{a}}$

$135.3 \pm 17.1^{\mathrm{a}}$

$157.4 \pm 12.1^{\mathrm{a} *}$

$59.2 \pm 7.1$

$46.8 \pm 10.0$

$43.4 \pm 7.2$

$52.9 \pm 5.9^{\mathrm{a}}$

$87.2 \pm 18.6^{\mathrm{a}}$

$106.5 \pm 13.1^{\mathrm{a} *}$ d14

$19.2 \pm 1.1$

$12.0 \pm 0.8^{\mathrm{a} *}$

$11.7 \pm 0.8^{\mathrm{a} *}$

$145.2 \pm 4.9^{\mathrm{b}}$

$157.4 \pm 16.7^{\mathrm{a}}$

$166.0 \pm 9.6^{\mathrm{a}}$

$75.5 \pm 8.4^{\mathrm{a}}$

$31.4 \pm 3.6^{*}$

$33.5 \pm 3.7^{*}$

$70.7 \pm 3.8^{\mathrm{b}}$

$114.2 \pm 16.2^{\mathrm{b}} *$

$127.8 \pm 11.4^{\mathrm{a} *}$

Means \pm SEM. ${ }^{a} P<0.05$ vs. d6 within each individual group; ${ }^{\mathrm{b}} P<0.05$ vs. $\mathrm{d} 6$ and $\mathrm{d} 10$ within each individual group; $* P<0.05$ vs. control. 

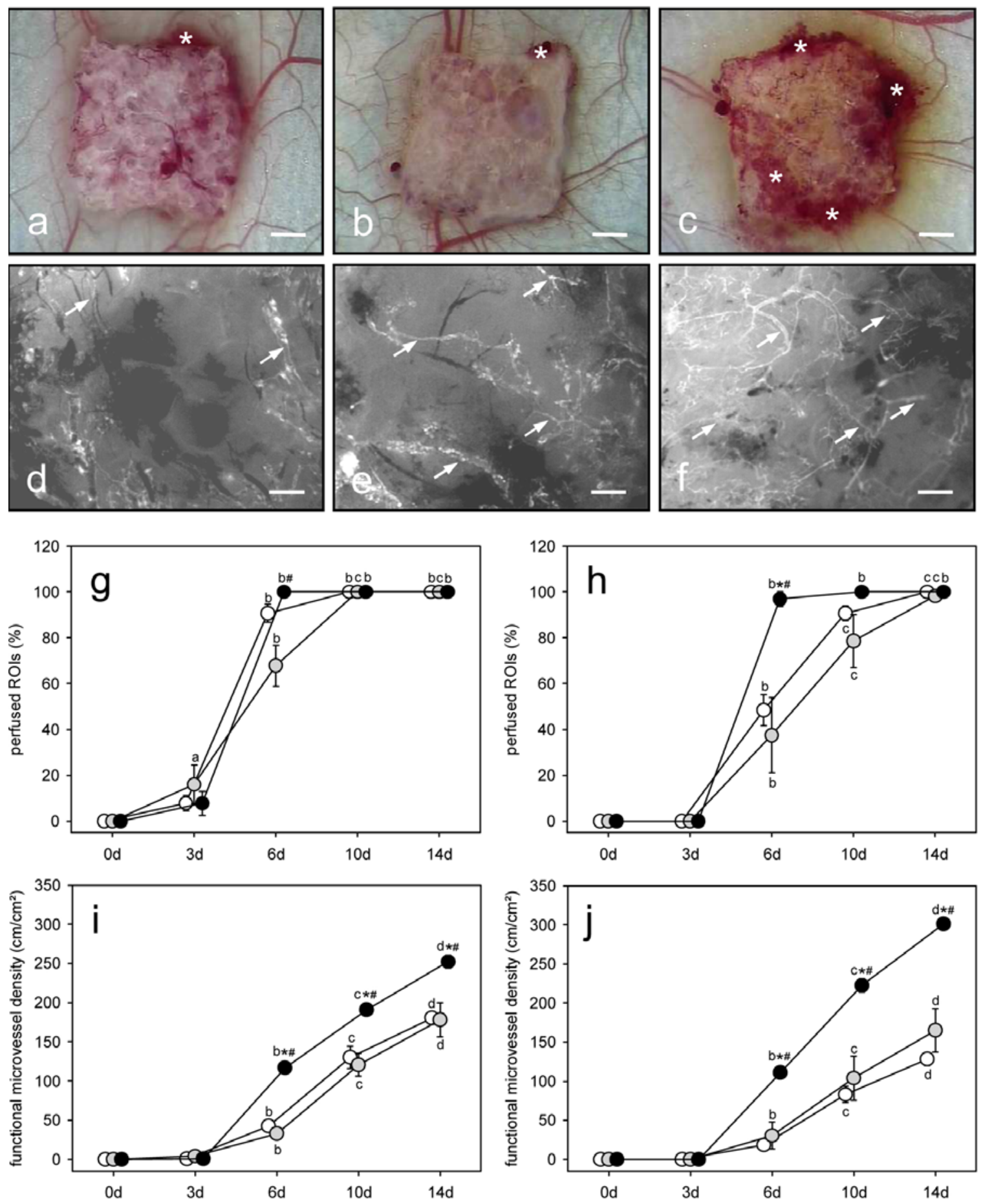

Fig. 3. (a-f): Stereo microscopy of in situ prevascularised tissue constructs at day 3 (a-c) and intravital fluorescence microscopy of blood perfused microvessels (arrows) within their centre at day 10 (d-f) after implantation into the dorsal skinfold chamber. The constructs were implanted into the chamber directly after embedding in Matrigel (b, e) or after 3 days of pre-cultivation in the extracellular matrix (c, f). Matrigel-free constructs (a, d) served as controls. Note that the construct, which was cultivated for 3 days in Matrigel, exhibits increased haemorrhages (c, asterisks) at day 3 after implantation into the dorsal skinfold chamber when compared to the other two groups (a, b, asterisks). Moreover, cultivation in Matrigel results in an improved vascularisation at day 10 (f vs. d and e). (d-f): Blue-light epi-illumination with contrast enhancement by 5\% FITC-labelled dextran 150,000 i.v.. Scale bars: (a-c) $=680 \mu \mathrm{m} ;(\mathbf{d}-\mathbf{f})=250 \mu \mathrm{m} .(\mathbf{g}-\mathbf{j})$ : Perfused ROIs $(\%)(\mathbf{g}, \mathbf{h})$ and functional microvessel density $(\mathbf{i}, \mathbf{j})$ within the border $(\mathbf{g}, \mathbf{i})$ and the centre zones $(\mathbf{h}, \mathbf{j})$ of in situ prevascularised tissue constructs after implantation into dorsal skinfold chambers, as assessed by intravital fluorescence microscopy and computer-assisted image analysis. The constructs were implanted into the chamber directly after embedding in Matrigel (grey circles) or after 3 days of pre-cultivation in the extracellular matrix (black circles). Matrigel-free constructs (white circles) served as controls. Means $\pm \mathrm{SEM}$. ${ }^{a} P<0.05$ vs. $0 \mathrm{~d}$ within each individual group; ${ }^{\mathrm{b}} \mathrm{P}<0.05 \mathrm{vs}$. $0 \mathrm{~d}$ and $3 \mathrm{~d}$ within each individual group; ${ }^{\mathrm{c}} \mathrm{P}<0.05 \mathrm{vs} .0 \mathrm{~d}, 3 \mathrm{~d}$ and $6 \mathrm{~d}$ within each individual group; ${ }^{\mathrm{d}} P<0.05$ vs. $0 \mathrm{~d}, 3 \mathrm{~d}, 6 \mathrm{~d}$ and $10 \mathrm{~d}$ within each individual group; $* P<0.05$ vs. control; ${ }^{\#} P<0.05$ vs. direct implantation after embedding in Matrigel. 

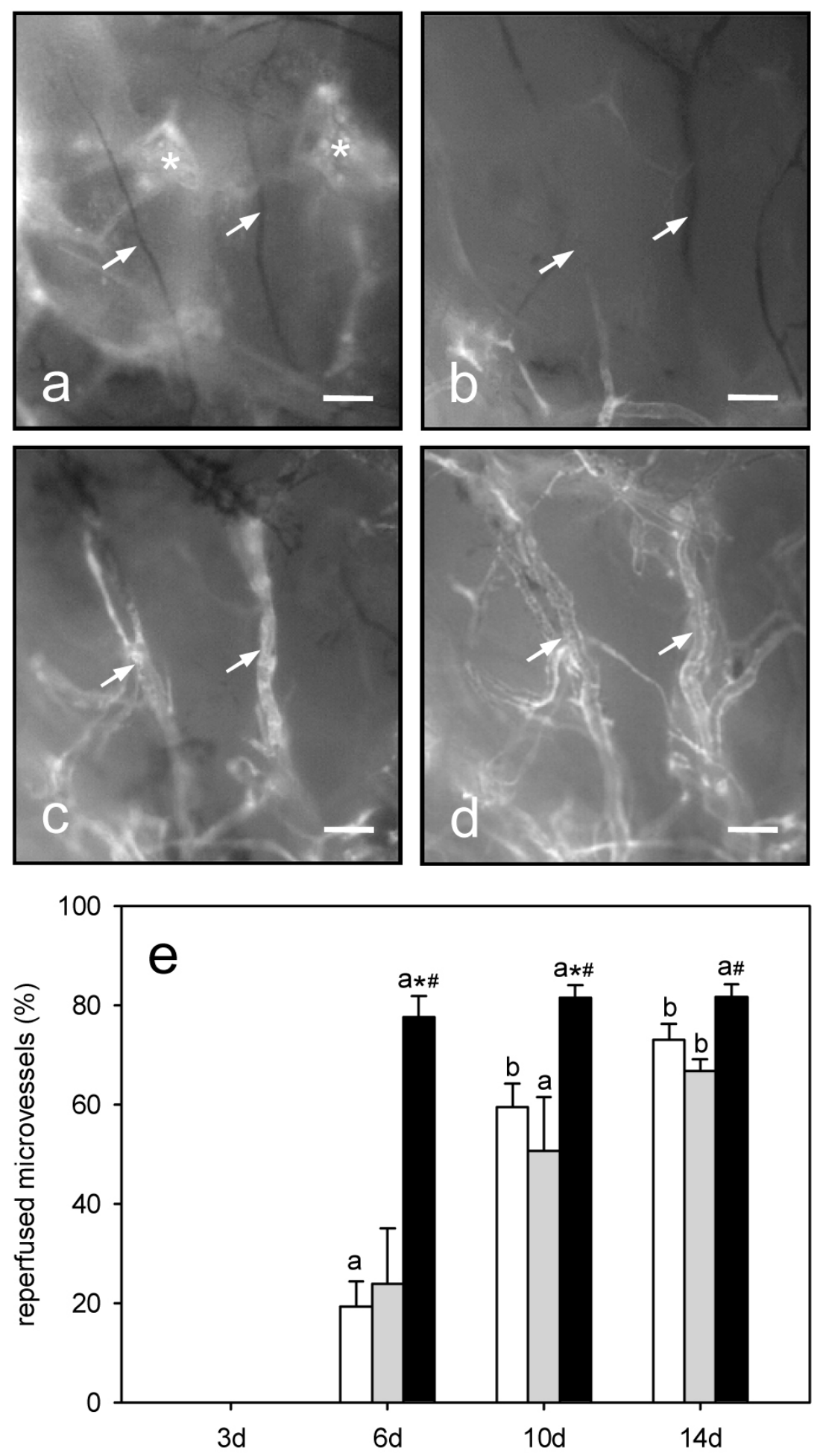

Fig. 4. (a-d): Intravital fluorescence microscopy of preformed microvessels (arrows) within the center of a Matrigelfree prevascularised tissue construct after implantation into the dorsal skinfold chamber. Fluorescence microscopy was performed directly after implantation into the dorsal skinfold chamber (a) as well as at day 6 (b), 10 (c) and 14 (d). Note that initially after implantation of the construct the preformed microvessels appear dark, indicating lack of FITC-dextran-labelled blood perfusion (a, b). At day 10 and 14 the microvessels are completely reperfused (c, d). This confirms that the preformed microvascular network within the in situ prevascularised tissue construct has become connected to blood vessels of the surrounding host tissue. Blue-light epi-illumination with contrast enhancement by 5\% FITC-labelled dextran 150,000 i.v.. Scaffold strands (a, asterisks) appear bright under high

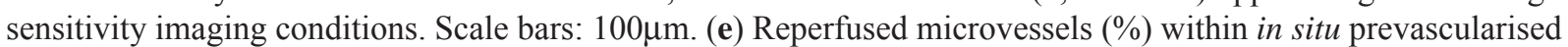
tissue constructs after implantation into the dorsal skinfold chamber, as assessed by intravital fluorescence microscopy and computer-assisted image analysis. The constructs were implanted into the chamber directly after embedding in Matrigel (grey bars) or after 3 days of pre-cultivation in the extracellular matrix (black bars). Matrigel-free constructs (white bars) served as controls. Means \pm SEM. ${ }^{a} P<0.05$ vs. $3 \mathrm{~d}$ within each individual group; ${ }^{\mathrm{b}} P<0.05 \mathrm{vs}$. $3 \mathrm{~d}$ and $6 \mathrm{~d}$ within each individual group; ${ }^{*} P<0.05$ vs. control; ${ }^{\#} P<0.05$ vs. direct implantation after embedding in Matrigel. 

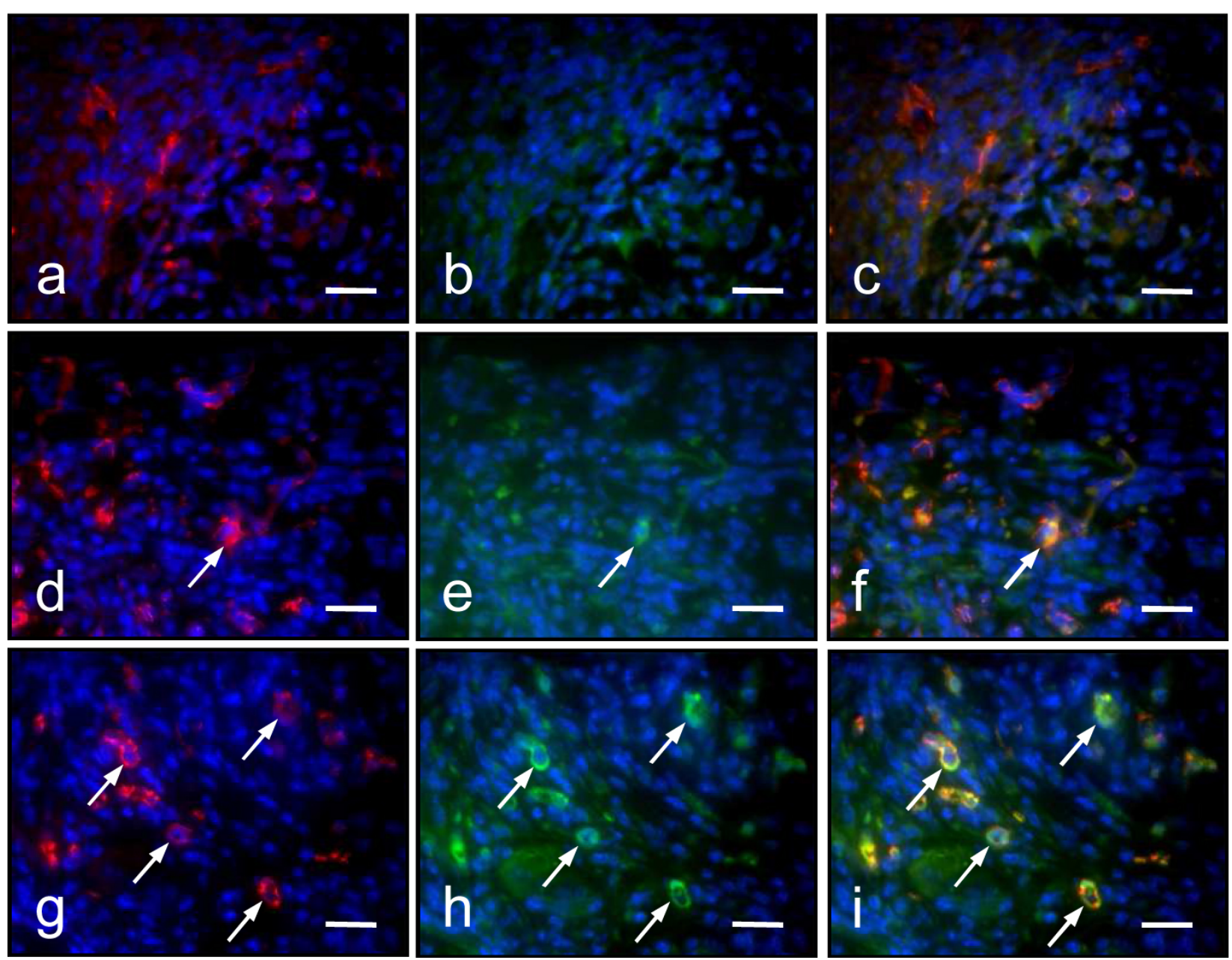

Fig. 5. Immunohistochemical characterization of microvessels in the border zone of in situ prevascularised tissue constructs at day 14 after implantation into the dorsal skinfold chamber. The constructs were implanted into the chamber directly after embedding in Matrigel (d-f) or after 3 days of pre-cultivation in the extracellular matrix (gi). Matrigel-free constructs (a-c) served as controls. Histological sections were stained with Hoechst to identify cell nuclei (a-i, blue), an antibody against CD31 for the detection of the microvascular endothelium (a, d, g, red), and an antibody against GFP for the detection of GFP-positive endothelial cells (b, e, h, green). c, $\mathbf{f}$ and $\mathbf{i}$ display merges of (a, b), (d, e) and (g, h), respectively. Note that the construct which was pre-cultivated for 3 days in Matrigel exhibits an increased number of GFP-positive microvessels (arrows) in the border zone when compared to the other two groups. Scale bars: $\mathbf{a - i}=26 \mu \mathrm{m}$.

increased throughout the 2-weeks observation period and were significantly elevated at day 14 when compared to that of the control group (Table 1).

\section{Inosculation of implanted tissue constructs}

In the present study we analyzed the inosculation of preformed microvascular networks within in situ prevascularised tissue constructs using intravital fluorescence microscopy (Fig. 4). Our quantitative analysis of reperfused microvessels within the implants revealed that pre-cultivation of tissue constructs in Matrigel markedly promoted the process of inosculation, as indicated by a significantly increased number of reperfused microvessels within these constructs between day 6 to day 10 when compared to the other two groups (Fig. 4). In fact, pre-cultivation of tissue constructs in Matrigel resulted already at day 6 in a 4-fold increased number of reperfused microvessels when compared with the two other groups.
We additionally analyzed the microvessels of the newly formed granulation tissue surrounding the tissue constructs at day 14 after implantation into the dorsal skinfold chamber (Fig. 5). Interestingly, we found that the constructs, which were pre-cultivated for 3 days in Matrigel, exhibited 33 $\pm 6 \%$ GFP-positive microvessels in their border zones. In contrast, non-cultivated Matrigelembedded and Matrigel-free control constructs presented with a significantly lower number of GFP-positive microvessels of $11 \pm 4 \%$ and $9 \pm 5 \%(p<0.05)$, respectively.

\section{Discussion}

In tissue engineering, the in vitro or in situ generation of microvascular networks offers the promising opportunity to rapidly establish a life-sustaining blood supply to implanted tissue constructs by the process of inosculation (Laschke et al., 2009). We herein demonstrate that this 
process can markedly be accelerated by pre-cultivation of prevascularised tissue constructs in an angiogenic extracellular matrix before implantation.

In our study, we cultivated in situ prevascularised tissue constructs in Matrigel. Due to its content of angiogenic growth factors, this extracellular matrix promotes the development of new blood vessels. Accordingly, Matrigel has been used for many years for the in vitro and in vivo analysis of angiogenesis and the identification of angiogenic inhibitors and stimulators (Kleinman and Martin, 2005). Moreover, incorporation of biomaterials into Matrigel has recently been shown to improve their vascularisation after implantation (Laschke et al., 2008b; Strieth et al., 2010). Because Matrigel is isolated from a poorly differentiated mouse chondrosarcoma, it may, however, not be the first choice for the modification of clinically applied tissue constructs. Nonetheless, we used it for our experiments, because its composition and angiogenic activity is well known and, thus, it ideally served as a standardized extracellular matrix for the present proof of principle study. A good alternative for a clinical application may be platelet rich plasma (PRP). This gel bears the major advantage that it can easily be generated from a patient's blood sample and, thus, exhibits an excellent biocompatibility (Eppley et al., 2006). Moreover, similar to Matrigel PRP contains a combination of different angiogenic growth factors that may ideally promote angiogenesis and external inosculation of pre-cultivated tissue constructs.

Our data show that Matrigel-embedded prevascularised tissue constructs exhibited a significantly increased functional capillary density at the border and center zones between day 6 and 14 after implantation into the dorsal skinfold chamber of recipient animals when compared to Matrigel-free controls. However, this was only the case for implants, which had been pre-cultivated for 3 days in Matrigel and not for those, which were directly transferred into the chamber after embedding in the extracellular matrix. Thus, the improved vascularisation of Matrigelembedded tissue constructs cannot simply be attributed to the angiogenic activity of the matrix itself, but may rather be due to the stimulation of vascular sprouting from the tissue constructs into the Matrigel during in vitro cultivation. Accordingly, we detected in vitro CD31/GFPpositive endothelial cells, which were migrated into the Matrigel margin surrounding the tissue constructs. Moreover, we observed large haemorrhages around the tissue constructs at day 3 after implantation into the dorsal skinfold chamber, which may result from micro-bleedings out of the fragile preformed sprouts. Our immunohistochemical analyses further revealed that the constructs, which were pre-cultivated for 3 days in Matrigel, exhibited significantly more GFP-positive microvessels in their border zone when compared to the other two groups. Taken together, all these results indicate that the pre-cultivation of the tissue constructs in Matrigel promoted external inosculation, resulting in a markedly accelerated restoration of blood perfusion in their preformed microvascular networks after implantation. In line with this interpretation microvessels in Matrigel-cultivated tissue constructs exhibited a significantly increased centreline velocity at day 10 when compared to controls.

For the generation of in situ prevascularised tissue constructs we used porous nano-size hydroxyapatite particles/poly(ester-urethane) composite scaffolds with a size of $\sim 3 \times 3 \times 1 \mathrm{~mm}$. Thus, the scaffolds were rather small and may not reflect the dimensions, which are needed in the clinical setting to fill up large tissue defects. In fact, it may probably not be possible to keep a thick threedimensional tissue construct in culture viable for several days, because the diffusion distance for oxygen and the culture medium to the cells in the centre of the construct may be too large to guarantee cell survival. However, this issue may be overcome by creating and cultivating several small prevascularised tissue constructs, which subsequently are implanted together into a large host defect site.

Interestingly, we found that microvessel diameters of implanted tissue constructs in both Matrigel groups progressively decreased, whereas the microvessel diameters in Matrigel-free control implants remained constant throughout the observation period of 14 days. This finding is most probably due to the fact that Matrigel does not only contain growth factors, which initiate angiogenesis, such as vascular endothelial growth factor (VEGF) (Yancopoulos et al., 2000), insulin-like growth factor I (IGF-1) (Beckert et al., 2006) and epidermal growth factor (EGF) (van Cruijsen et al., 2005), but also the subsequent maturation of the newly formed blood vessels, including platelet-derived growth factor (PDGF) (Jain, 2003) and transforming growth factor (TGF)- $\beta$ (Richardson et al., 2001). This maturation is associated with the stabilization of the vessel wall by recruitment of perivascular smooth muscle cells and is typically characterized by a decrease of microvessel diameters (Laschke et al., 2002; Gerhardt and Betsholtz, 2003). The small diameters and the early onset of high centreline velocities in microvessels of the Matrigel-cultivated tissue constructs may have further contributed to their improved vascularisation by shear stress-induced angiogenesis (Brown and Hudlika, 2003; Resnick et al., 2003).

In summary, we herein provide the proof of principle that the establishment of a blood supply to prevascularised tissue constructs can be accelerated by their pre-cultivation in an angiogenic extracellular matrix, promoting external inosculation of the preformed microvascular networks after implantation into the host tissue. This easy approach may contribute to a better survival of cells within tissue constructs and, thus, may increase the success rates of future tissue engineering applications.

\section{Acknowledgements}

We are grateful for the excellent technical assistance of Janine Becker. This work was supported by the CRC Homburg/AO Foundation. 


\section{References}

Baker M, Wayland H (1974) On-line volume flow rate and velocity profile measurement for blood in microvessels. Microvasc Res 7: 131-143.

Beckert S, Farrahi F, Perveen Ghani Q, Aslam R, Scheuenstuhl H, Coerper S, Königsrainer A, Hunt TK, Hussain MZ (2006) IGF-I-induced VEGF expression in HUVEC involves phosphorylation and inhibition of poly(ADP-ribose)polymerase. Biochem Biophys Res Commun 341: 67-72.

Brown MD, Hudlicka O (2003) Modulation of physiological angiogenesis in skeletal muscle by mechanical forces: involvement of VEGF and metalloproteinases. Angiogenesis 6: 1-14.

Capla JM, Ceradini DJ, Tepper OM, Callaghan MJ, Bhatt KA, Galiano RD, Levine JP, Gurtner GC (2006) Skin graft vascularisation involves precisely regulated regression and replacement of endothelial cells through both angiogenesis and vasculogenesis. Plast Reconstr Surg 117: 836-844.

Eppley BL, Pietrzak WS, Blanton M (2006) Plateletrich plasma: a review of biology and applications in plastic surgery. Plast Reconstr Surg 118: 147e-159e.

Gerhardt H, Betsholtz C (2003) Endothelial-pericyte interactions in angiogenesis. Cell Tissue Res 314: 15-23.

Jain RK (2003) Molecular regulation of vessel maturation. Nat Med 9: 685-693.

Kleinman HK, Martin GR (2005) Matrigel: basement membrane matrix with biological activity. Semin Cancer Biol 15: 378-386.

Laschke MW, Menger MD, Vollmar B (2002) Ovariectomy improves neovascularisation and microcirculation of freely transplanted ovarian follicles. J Endocrinol 172: 535-544.

Laschke MW, Harder Y, Amon M, Martin I, Farhadi J, Ring A, Torio-Padron N, Schramm R, Rücker M, Junker D, Häufel JM, Carvalho C, Heberer M, Germann G, Vollmar B, Menger MD (2006) Angiogenesis in tissue engineering: breathing life into constructed tissue substitutes. Tissue Eng 12: 2093-2104.

Laschke MW, Rücker M, Jensen G, Carvalho C, Mülhaupt R, Gellrich NC, Menger MD (2008a) Improvement of vascularisation of PLGA scaffolds by inosculation of in situ-preformed functional blood vessels with the host microvasculature. Ann Surg 248: 939-948.

Laschke MW, Rücker M, Jensen G, Carvalho C, Mülhaupt R, Gellrich NC, Menger MD (2008b) Incorporation of growth factor containing Matrigel promotes vascularisation of porous PLGA scaffolds. J Biomed Mater Res A 85: 397-407.

Laschke MW, Vollmar B, Menger MD (2009) Inosculation: connecting the life-sustaining pipelines. Tissue Eng Part B Rev 15: 455-465.

Laschke MW, Strohe A, Menger MD, Alini M, Eglin $\mathrm{D}$ (2010) In vitro and in vivo evaluation of a novel nanosize hydroxyapatite particles/poly(ester-urethane) composite scaffold for bone tissue engineering. Acta Biomater 6: 2020-2027.

Lokmic Z, Mitchell GM (2008) Engineering the microcirculation. Tissue Eng Part B Rev 14: 87-103.
Lovett M, Lee K, Edwards A, Kaplan DL (2009) Vascularisation strategies for tissue engineering. Tissue Eng Part B Rev 15: 353-370.

Menger MD, Laschke MW, Vollmar B (2002) Viewing the microcirculation through the window: some twenty years experience with the hamster dorsal skinfold chamber. Eur Surg Res 34: 83-91.

Motoike T, Loughna S, Perens E, Roman BL, Liao W, Chau TC, Richardson CD, Kawate T, Kuno J, Weinstein BM, Stainier DY, Sato TN (2000) Universal GFP reporter for the study of vascular development. Genesis 28: 75-81.

Nicosia RF, Ottinetti A (1990) Modulation of microvascular growth and morphogenesis by reconstituted basement membrane gel in three-dimensional cultures of rat aorta: a comparative study of angiogenesis in matrigel, collagen, fibrin, and plasma clot. In Vitro Cell Dev Biol 26: 119-128.

Resnick N, Yahav H, Shay-Salit A, Shushy M, Schubert S, Zilberman LC, Wofovitz E (2003) Fluid shear stress and the vascular endothelium: for better and for worse. Prog Biophys Mol Biol 81: 177-199.

Richardson TP, Peters MC, Ennett AB, Mooney DJ (2001) Polymeric system for dual growth factor delivery. Nat Biotechnol 19: 1029-1034.

Rouwkema J, Rivron NC, Van Blitterswijk CA (2008) Vascularisation in tissue engineering. Trends Biotechnol 26: 434-441.

Strieth S, Weger T, Bartesch C, Bittmann P, Stelter K, Berghaus A (2010) Biocompatibility of porous polyethylene implants tissue-engineered by extracellular matrix and VEGF. J Biomed Mater Res A 93: 1566-1573.

Tremblay PL, Hudon V, Berthod F, Germain L, Auger FA (2005) Inosculation of tissue-engineered capillaries with the host's vasculature in a reconstructed skin transplanted on mice. Am J Transplant 5: 1002-1010.

Van Cruijsen H, Giaccone G, Hoekman K (2005) Epidermal growth factor receptor and angiogenesis: Opportunities for combined anticancer strategies. Int $\mathrm{J}$ Cancer 117: 883-888.

Yancopoulos GD, Davis S, Gale NW, Rudge JS, Wiegand SJ, Holash J (2000) Vascular-specific growth factors and blood vessel formation. Nature 407: 242-248.

\section{Discussion with Reviewers}

Reviewer I: How do you think to use these proof-ofprinciple results in a clinical setting? Have you considered the possibility to use matrices of different origin for preculturing implants?

Authors: In the present proof-of-principle study we show that external inosculation of prevascularised tissue constructs is promoted by pre-cultivation of the implants in an angiogenic extracellular matrix. For this purpose, we cultivated in situ prevascularised tissue constructs in Matrigel, which is a well-defined extracellular matrix. However, Matrigel may not be the first choice to translate our work into the clinic, because it is obtained from a mouse chondrosarcoma. A good alternative for the clinical application of our findings may be the use of platelet rich plasma (PRP). This gel bears the major advantage that it 
can easily be generated from a patient's blood sample and thus exhibits an excellent biocompatibility. Moreover, similar to Matrigel, PRP contains a combination of different angiogenic growth factors that may ideally promote angiogenesis and external inosculation of pre-cultivated tissue constructs in a clinical setting.

For the generation of prevascularised tissue constructs, we used porous nano-size hydroxyapatite particles/ poly(ester-urethane) composite scaffolds, because we previously found that these scaffolds exhibit a good in vivo biocompatibility. However, we feel that the principle shown in the present study is transferable to any other type of scaffold, which may be used for clinical tissue engineering applications.

Reviewer II: Since external inosculation implies the ingrowth of transplanted vessels into the host, in clinical models that can only be achieved by means of autologous transplantation and thus has limited implications. Please comment.

Authors: We agree with the reviewer that the principle shown in the present study is only transferable to the clinical setting by means of autologous transplantation of prevascularised tissue constructs to prevent graft rejection. However, we feel that this does not necessarily limit its applications. For instance, it may be possible to prevascularise a tissue construct in a first step at an easily accessible implantation site of the patient, such as the subcutaneous tissue. The prevascularised tissue construct may then be carefully excised and pre-cultivated in an extracellular matrix before implantation into the final host defect site.

Reviewer II: Additionally the size of the scaffolds should be discussed. The size used by the author is suitable to demonstrate the concept of external inosculation and analyze blood flow and other parameters, but successful inosculation of scaffolds with a thickness of $1 \mathrm{~mm}$ is of limited clinical relevance. The authors should also discuss the need to vascularise true three-dimensional scaffolds of relevant dimension. It would certainly be interesting to see the effect of pre-cultivation there.
Authors: We agree with the reviewer that the size of the scaffolds used in the present study was rather small and may not reflect the dimensions, which are needed in the clinical setting to fill up large tissue defects. On the other hand, there may be the problem that it is probably not possible to keep a rather thick three-dimensional tissue construct in culture viable for several days, because the diffusion distance for oxygen and the culture medium to the cells in the centre of the construct may be too large to guarantee cell survival. However, this issue may be overcome by creating and cultivating several small prevascularised tissue constructs, which subsequently are implanted together into a large host defect site.

Reviewer II: Also, a second control group compromising of implanted constructs without prevascularisation would have been interesting concerning internal inosculation, blood flow and vascularisation, as well.

Authors: We agree with the reviewer that it would be interesting to compare prevascularised and nonprevascularised tissue constructs in terms of internal inosculation, blood perfusion and vascularisation. However, we already made this comparison in a previous study (Laschke et al., 2008a, text reference). In this previous study we could demonstrate that prevascularised PLGA scaffolds exhibit a significantly improved vascularisation due to inosculation after implantation when compared to non-prevascularised control scaffolds. However, we also found that the prevascularised constructs did not exhibit an adequate blood perfusion during the very first days after implantation. Accordingly, we decided to focus in the present study only on prevascularised scaffolds with the aim to accelerate their inosculation by pre-cultivation in Matrigel.

In addition, the inclusion of a group of nonprevascularised scaffolds would make it necessary to perform additional control groups of non-prevascularised scaffolds, which are embedded in Matrigel with and without pre-cultivation before implantation. This study design, however, would exceed the scope of the present study. 